\title{
Énumération des Cestodes du plancton et des Invertébrés marins
}

\author{
$9^{e}$ Contribution (1)
}

\author{
par Robert-Ph. DOLLFUS † \\ Laboratoire de Zoologie (Vers) associé au C.N.R.S., \\ Muséum national d'Histoire naturelle, 43 rue Cuvier, F 75231 Paris Cedex 05.
}

\section{Résumé.}

Dans cette $9^{\circ}$ Contribution, on trouvera la mention de Cestodes trouvés: A chez des Lamellibranches, в chez des Gastropodes, c chez des Ptéropodes, D chez des Céphalopodes, E chez des Crustacés.

\section{Summary.}

This 9th Contribution concerns Cestodes obtained from: A Lamellibranchiata, B Gastropoda, c Pteropoda, D Cephalopoda, E Crustacea.

\section{A. - Chez des Lamellibranches}

\section{A-1. Heterodonta Veneracea.}

Chez Meretrix casta (Chemnitz, 1782) de la côte de Madras, Lothar W. Reimer a trouvé des larves de trois espèces de Cestodes :

1. Larve du groupe Scolex pleuronectis O.F.M., dans la musculature. Longueur totale $1900 \mu$, largeur $485 \mu$ dans la région postérieure, $650 \mu$ au niveau des bothridies.

(1) Les Contributions précédentes ont été publiées dans les Ann. Parasit. hum. comp. en 1923, 1924, 1926, 1929, 1931, 1964, 1967.

$\dagger(1887-1976)$. 
Les bothridies mesurent 318-324 $\mu$ de long sur 190-200 $\mu$ de large; il y a une énorme ventouse accessoire (135-150 $\mu$ de diamètre). Le myzorhynchus est très petit (40-48 $\mu$ de diamètre). L. Reimer (1975, pp. 6-7, fig. 4) a estimé, dubitativement, qu'il s'agissait peut-être du genre Marsupiobothrium S. Yamaguti 1952, dont les bothridies ont une forme voisine. Reimer a estimé que ce plérocercoïde avait une évidente ressemblance avec celui, parasite de Sepia filliouxi Lafont, décrit par R.-Ph. Dollfus (1974, pp. 341342, fig. 12-13), qui a un myzorhynchus proportionnellement d'un plus grand diamètre.

2. Larve d'Echeneibothrium sp., deux exemplaires dans la musculature. Longueur : 2140 et $2520 \mu$; largeur : 314 et $294 \mu$. Bothridies 190-204 $\times 103-110 \mu$. Myzorhynchus 60-72 $\times 82-99 \mu$. Les bothridies ont environ 22 loculi. Ayant décrit cette larve, L. Reimer (1975, p. 8, fig. 6) la compare aux larves d'Echeneibothrium décrites ou mentionnées par W.S. Hunter (1950), C.E. Mc Ginitie et N. Mc Ginitie (1945), L. H. Hyman (1951), A. K. Sparks et K. K. Chew (1966) chez divers invertébrés et aussi avec un parasite d'Eupagurus que j'ai désigné sous le nom «Plerocercoïdes: Echeneibothrium (gen. ?) mouchetae nom. provis. » et dont l'attribution à Echeneibothrium est dubitative (voir R. Ph. Dollfus, 1931, pp. 553-560, fig. 1-7).

3. Larve de Tylocephalum sp., trouvée aussi chez Turritella attenuata Reeve, Mactra mera Reeve, Arca inaequivalvis Bruguière. Reimer (1975, pp. 9-10) a réparti les larves de Tylocephalum qu'il a observées en deux groupes; celles parasitant Meretrix casta (Chemnitz) ont été classées dans le deuxième groupe. Dans ce groupe, la longueur des larves varie de 872 à $5420 \mu$, la largeur de 139 à $990 \mu$; leur extrémité antérieure ne présente pas le puissant myzorhynchus typique pour le genre, mais elle est plutôt pointue et, dans quelques cas, déprimée et tronquée. Les plus grands individus étaient ceux parasitant Mactra et Meretrix. Reimer rappelle que, chez des Téléostéens de Madras, des larves attribuées à Tylocephalum ont été décrites par C. K. Subhapradha (1948) (2). Celles considérées par ce dernier comme au deuxième stade avaient l'habitus caractéristique de celles du deuxième groupe récoltées chez les mollusques des quatre espèces mentionnées ci-dessus.

\section{A-2. Heterodonta Mactracea.}

Chez des Mactra mera Reeve de la côte de Madras, L. W. Reimer (1975, p. 10) a trouvé la larve d'un Tylocephalum sp. La même larve parasitait aussi Turritella attenuata Reeve, Meretrix casta (Chemnitz), Arca inaequivalvis Bruguière, de la même localité (vide supra).

\section{A-3. Heterodonta Tellinacea.}

Chez Donax scortum L. de la côte de Madras, L. W. Reimer $(1975$, p. 6) a trouvé une larve du type Scolex pleuronectis O.F.M., montrant les mêmes caractéristiques que celle parasitant Turritella attenuata Reeve, de la même localité (vide infra).

(2) Je n'ai pas pu consulter l'ouvrage de cet auteur (Thèse non publiée de l'Université de Madras). 


\section{A-4. Taxodonta Archacea.}

Chez des Arca inaequivalvis Bruguière, de la côte de Madras, L. W. Reimer (1975, p. 10) a trouvé la larve d'un Tylocephalum sp. Il a trouvé la même larve chez Turritella attenuata Reeve, Meretrix casta (Chemnitz), Mactra mera Reeve, de la même localité (vide supra).

\section{A-5. Anisomyaria Ostracea.}

J. A. Quick jr (1971, p. 143), relate avoir trouvé périodiquement des corpuscules d'un diamètre de 200-700 $\mu$, de forme circulaire à ovoïde, entourés de fibres élastiques, dans le tissu conjonctif et parfois le tissu de Leydig des Crassostrea virginica (Gmelin) de l'Apalachicola Bay (Florida). Ces corpuscules ont été tentativement attribués à des larves de Tylocephalum sp., mortes ou en voie de résorption (Quick, 1971. p. 188, pl. color. V-3, fig. A, larve de Tylocephalum encapsulée dans de nombreuses couches de fibres élastiques dans le tissu conjonctif, près de l'estomac). Les huîtres ainsi parasitées étaient dans une eau de mer dont la température avait été élevée expérimentalement à $35^{\circ} \mathrm{C}$.

Toutefois, des huîtres de Chortawhatcher Bay (Florida) contenaient des larves de Cestode encore vivantes (ibid., pp. 143, 145, fig. V-11, A, B et pl. color. V-3, fig. B), avec épithelium cilié et myzorhynchus ; elles étaient situées sous l'épithelium intestinal, encapsulées par de nombreuses couches de fibres élastiques, dans du tissu conjonctif, montrant une réaction immunitaire locale. Pour ces larves, Quick estima que Crassostrea virginica (Gmelin) était un «improper intermediate host ».

Erik Rifkin et Thomas C. Cheng (1969, pp. 166-167) ont étudié au microscope électronique la structure fine du tégument de la larve de Tylocephalum sp. (Lecanicephala) parasite interne de mollusques. Ils ont reconnu que ce tégument est délimité en dedans par une couche de matériaux fibreux, tandis que la surface externe est bordée par une membrane plasmatique. Des mitochondries et des vésicules de densité électronique variable sont présents dans le tégument. Des expansions digitiformes de la couche basale se projettent dans le tégument de la surface interne. De grandes épines ayant une densité électronique sont bordées chacune par une membrane plasmatique externe s'étendant au dehors, à partir de la périphérie du tégument. Se projetant de la surface externe du tégument, il y a des microvilli comprenant des régions bien délimitées, la région la plus externe d'entre elles est bordée par une membrane plasmatique. Ces projections cytoplasmiques ne se présentent pas comme la région baculiforme proximale caractéristique se terminant par un apex à densité électronique, comme cela a été trouvé associé avec les téguments du cestode par d'autres chercheurs. Secondairement, des projections cytoplasmiques latérales s'élèvent des microvilli primaires. Des vésicules limitées par une membrane sont trouvées intimement associées aux microvilli. La formation et la fonction de celles-ci restent spéculatives, disent Rifkin et Cheng.

En une autre publication, Erik Rifkin (1969, p. 4432 B) a relaté la formation et la structure de la capsule formée par l'huître, Crassostrea virginica (Gmelin) pour l'isolement de la larve de Tylocephalum sp. Il a étudié la structure de l'interface hôte-parasite. Le stade initial du développement de la capsule apparaît, dit Rifkin, lorsque le 
parasite envahisseur comprime les cellules de Leydig qui l'entourent. Cela est suivi par un épaississement du matériel intercellulaire entre les cellules de Leydig et l'infiltration par des leucocytes. Finalement, des cellules fibroblastiques se montrent dans la région la plus interne de la capsule et le matériel intercellulaire devient manifestement fibreux. Ces fibres réticulaires et la matrice dans laquelle elles sont englobées comprennent des glucoprotéines et (ou) des mucoprotéines, ainsi que des mucopolysaccharides neutres. L'étude au microscope électronique des constituants de la capsule a révélé trois types de cellules et deux types de fibres extracellulaires, dont la microstructure a été décrite par Rifkin. En outre, celui-ci a reconnu que la concentration en protéines de l'hémolymphe a une décroissance significative chez les huîtres placées pendant un certain temps dans les confins environnant les individus parasités par le Tylocephalum.

\section{B. - Chez des Gastropodes}

\section{B-1. Stenoglossa Buccinacea.}

Chez Bullia melanoides (Deshayes) de la côte de Madras, L. W. Reimer (1975) a trouvé des larves d'Echinobothrium, de Polypocephalus et de Christianella.

$1^{\circ}$ pp. 3-4, fig. 1. Echinobothrium lateroporum Subhapradha, 1948 (3), représenté par deux formes, l'une : Echinobothrium lateroporum lateroporum, à crochets incolores, l'autre: Echinobotrium lateroporum nigracanthum L. W. Reimer sub. sp., à crochets noirs.

Dimensions pour la forme à crochets incolores : 490-1 $790 \times 220-525 \mu$, larve incluse 276-570 $\times$ 80-155 $\mu$. Longueur des bothridies 159-264 $\mu$; crochets grands 70-118 $\mu$, moyens $56-90 \mu$, petits 10-14 $\mu$. Le nombre des grands crochets atteint 40 . Il y a 30 grands crochets disposés selon leur longueur, avec une base d'implantation commune et de chaque côté 15 très petits crochets.

Dimensions pour la forme à crochets noirs, sur le vivant: kystes 1650 $2000 \times 600 \mu$, longueur des bothridies $350 \mu$, longueur des crochets $100,73 \mu$. Après fixation : kystes $970 \times 368 \mu$, larve incluse $318 \times 107 \mu$; longueur des bothridies $180 \mu$.

L. W. Reimer (1975, p. 4) estime que la seule espèce adulte qui semble lui correspondre est Echinobothrium musteli Pintner, dont Subhapradha a séparé lateroporum.

$-2^{\circ}$ pp. 8-9, fig. 7. Sous l'appellation «Polypocephalus spec., larva », Reimer a décrit une larve du genre Polypocephalus M. Braun, 1878 (= Parataenia E. Linton, 1889) (famille Lecanocephalidae). Cette larve allongée, étroite, portait 14 tentacules non branchus, longs d'environ $130 \mu$, épais distalement de $25 \mu$, prenant naissance dans une dépression. La longueur était de $1000 \mu$ et la largeur de $284 \mu$ au niveau

(3) Il a déjà été question d'Echinobothrium lateroporum, C. K. Subhapradha 1948 dans une de mes précédentes publications (voir R.-Ph. Dollfus 1964, p. 338). 
de la mi-longueur. La région antérieure du corps se distinguait nettement par la présence de nombreux noyaux dans le parenchyme, vers le premier tiers, la région postérieure était longue de $665 \mu$. Une révision du genre a été publiée par Subhapradha (1951), qui a admis 11 espèces dans les eaux indiennes. Après comparaison avec ces espèces, Reimer a estimé qu'il s'agissait probablement de Polypocephalus lintoni Subhapr., 1951.

- $3^{\circ}$ pp. 12-14, fig. 11-14: larve de Christianella sp. Il s'agit d'un plerocercus encore inclus dans son blastocyste, considéré par Reimer comme plérocercoïde. Longueur 917-990 $\mu$, largeur 328-354 $\mu$. Bothridies $234 \times 183 \mu$. Pars vaginalis (excl. Pars bothridialis) longueur 341-360 $\mu$; dans cette partie, la gaine des proboscis est fortement annelée. Pars bulbosa, longueur 370-396 $\mu$. Largeur des bulbes 60-77 $\mu$. Pars postbulbosa relativement courte, 68-80 $\mu$. Les proboscis évaginés ont une longueur maximum de $666 \mu$, ils sont pourvus d'un renflement métabasal, et le rétracteur s'insère au fond des bulbes. Crochets des rangées basales, environ 7,5 $\mu$, des métabasales, $8 \mu$; autres crochets, longueur entre 4 et $6 \mu$. La même larve, incomplètement développée, a été trouvée chez Thais rudolphi (Lamarck), il en sera question plus loin à propos des parasites des Stenoglossa muricacea.

K. A. Hamilton et J. E. Byram (1974, p. 20) relatent que, dans la région d'Alligator Harbour (Florida), on trouve communément la larve plérocercoïde d'un Tétraphyllide dans l'estomac de plusieurs espèces de Gastropodes: 5 espèces de Stenoglossa Buccinacea : Fasciolaria tulipa (L.), Fasciolaria hunterica (Perry), Pleuroploca gigantea (Kiener), Melongena coronata (Gmelin), Busycon spiratum (Lamarck) et une espèce de Mesogastropoda Naticacea : Polinices duplicatus (Say). Il a été question antérieurement des larves trouvées chez Fasciolaria tulipa (L.) par F.-E. Friedl et J.-L. Simon (1970) et chez Melongena coronata (Gmelin) par J.-D. Regan (1963) (voir R.-Ph. Dollfus, 1974, p. 392).

Les plérocercoïdes récoltés dans l'estomac des Fasciolaria tulipa (L.) ont été l'objet de recherches physiologiques in vitro par K. A. Hamilton et J. E. Byram (1974, pp. 20-27, fig. 1-20) sur l'effet de l'urée sur la morphologie et la biochimie de ces larves.

Sous l'effet de l'urée, la ventouse apicale disparaît et les bothridies quadriloculaires deviennent triloculaires avec ventouse accessoire. Il apparaît un bourrelet musculaire et une paire de crochets bifurqués par bothridium, ce qui justifiait l'attribution de ces larves à la famille Onchobothriidae et au genre Acanthobothrium P. J. Van Beneden, 1849.

Ces auteurs ont mis en évidence la présence d'une protéine dans les crochets de cet Acanthobothrium. L'adulte correspondant n'a pas été précisé spécifiquement.

L'action de l'urée in vitro sur le plérocercoïde n'implique pas, d'après ces auteurs que l'urée soit le seul stimulus pour la croissance et le développement dans l'hôte définitif ; ils rappellent que, chez un Acanthobothrium sp. adulte, Simmons (1960) et d'autres auteurs ont reconnu l'absence d'activité uréasique et conclu que, pour cet Acanthobothrium sp., l'urée est un composé métaboliquement inactif. 


\section{B-2. Stenoglossa Muricacea.}

Chez Thais rudolphi (Lamarck) de la côte de Madras, diverses larves de Cestodes ont été trouvées par L. W. Reimer (1975) :

$-1^{\circ}$ pp. 4-5, fig. 2, Scolex pleuronectis O.F.M., à bothridies uniloculaires et grande ventouse apicale. Dimensions: 230-394 $\times$ 125-139 $\mu$. Myzorhynchus 58$60 \times 70-72 \mu$. Bothridies 71-82 $\times$ 44-64 $\mu$.

$-2^{\circ}$ p. 9, fig. 8: Tylocephalum sp. L.W. Reimer a distingué deux groupes parmi les larves de Lecanicephalidae qu'il a récoltées chez des mollusques de la côte de Madras. Celles parasitant Thais rudolphi (Lamarck) appartiennent au premier groupe. Longueur 127-218 $\mu$, largeur 83-131 $\mu$. Bothridies non observables. Myzorhynchus compact, le plus souvent rétracté dans le parenchyme, aussi protracté, mesurant 42-68 $\times$ 44-87 $\mu$. En arrière du myzorhynchus, des amas de noyaux de cellules.

- $3^{\circ}$ pp. 10-12, fig. 9-10: «Tetrarhynchobothrium spec., larva ». Longueur $4170 \mu$, bothridies patelliformes avec un bourrelet marginal. Gaines des proboscis peu ondulées. Pars bothridialis longue de $370 \mu$. Bothridies longues de $410 \mu$, Pars vaginalis antérieurement dépourvue de cellules glandulaires, longue de $450 \mu$, suivie par une région longue de $870 \mu$ dont le parenchyme est rempli de cellules glandulaires jusqu'au niveau du bord antérieur des bulbes. Les bulbes mesurent $656 \times 120 \mu$. Le rétracteur s'insère au fond des bulbes. Pars postbulbosa, longueur $1824 \mu$, largeur maximum $605 \mu$. Largeur des proboscis 24-27 $\mu$. A la base, les crochets ont une base large de 6-7 $\mu$ et une longueur de 5-6 $\mu$. Il y a 8-9 crochets par rangée.

- $4^{\circ}$ pp. 12-13 Plerocercus de Christianella sp. encore contenu dans son blastocyste et incomplètement développé. Longueur $341 \mu$, largeur $242 \mu$ au niveau du scolex, $140 \mu$ en arrière. Pars bothridialis, longueur $115 \mu$; pars vaginalis $79 \mu$, pars bulbosa $119 \mu$, pars postbulbosa $28 \mu$. Les probocis avec leurs crochets ne sont pas encore complètement formés.

Reimer est d'avis que cette larve de Christianella pourrait, d'après ses proportions, correspondre à celle observée par J. Azariah (1968, pp. 439-440, fig. 1-2) à Madras, chez Branchiostoma lanceolatum Pallas, dans la région de l'intestin moyen.

Sous l'appellation «Scolex spec. (Myzophyllobothrium spec. larvae (?) », L. W. Reimer (1975, p. 7, fig. 5) a décrit un plérocercoïde de Tétraphyllide trouvé chez Murex trapa Roding de la côte de Madras. C'est une forme longue et étroite (longueur 900-925 $\mu$, largeur 103-107 $\mu$ ), avec ventouse apicale $(54-57 \times 57-59 \mu)$ et quatre bothridies latérales longues de 73-97 $\mu$, immédiatement en arrière de la ventouse apicale. Chaque bothridie présente antérieurement une ventouse; en arrière des bothridies s'insérent des appendices longs de 58-68 $\mu$. Reimer suppose que cette larve appartient peut-être au genre Myzophyllobothrium Shipley et Hornell, 1906, dont les adultes ont été trouvés à Ceylan, chez des Myliobatis et Aetobatis.

\section{B-3. Mesogastropoda Cerithiacea.}

Chez Turritella attenuata Reeve de la côte de Madras, L. W. Reimer (1975, p. 5, fig. 6) a trouvé une larve du type Scolex pleuronectis O.F.M. (dimensions $770 \times 132 \mu$ ) 
dont les bothridies montraient une indication de trilocularité ; la ventouse apicale était volumineuse (diamètre 52-63 $\mu$ ), les bothridies latérales mesuraient 124-139 $\times$ 35-55 $\mu$. La même larve a été récoltée par L. W. Reimer chez Donax scortum L. Chez le même hôte, Reimer (1975, p. 9) a trouvé la larve d'un Tylocephalum parasitant aussi Mactra mera Reeve (Vide supra).

\section{B-4. Mesogastropoda Ptenoglossa.}

Chez Janthina globosa Swainson de la côte de Madras, L. W. Reimer (1975, pp. 3 , 6) a trouvé une larve du type Scolex pleuronectis O.F.M. à bothridies uniloculaires. Dimensions: longueur 60-116 $\mu$, largeur 49-87 $\mu$. Diamètre des bothridies latérales 16-29 $\mu$. Ventouse apicale (myzorhynchus) 23-46 $\mu$. Reimer a rapproché les spécimens récoltés par lui chez Janthina de ceux récoltés chez le Cténophore Pleubrachia globosa Moser à Madras; il renvoie pour celui-ci à Anantaraman et Krishnaswamy.

\section{C. - Chez des Ptéropodes}

A. J. Slankis et G. G. Shevschenko (1974, p. 134) mentionnent Euclio pyramidata (L.) parmi les hôtes de Tétrarhynques larvaires dans le plancton du Pacifique tropical occidental.

\section{D. - Chez des Céphalopodes}

L. W. Reimer (1974, p. 1728 ) relate, en une note préliminaire, qu'il a trouvé: $1^{\circ}$ des larves de Dinobothrium et de Grillotia chez des Sepia officinalis L. de la mer de la Manche; $2^{\circ}$ des Scolex polymorphus O.F.M. unilocularis, bilocularis et trilocularis chez des Sepia officinalis L., Loligo vulgaris Lamarck, Ommastrephes sagittatus (Lamarck). Les lieux de récolte ne sont pas précisés (Mer de la Manche ou Rio de Oro ?).

T. Shimazu (1975, pp. 824, 827) rappelle que Nybelinia surmenicola Yô K. Okada in R.-Ph. Dollfus, 1929, sous sa forme préadulte est un parasite commun chez le Céphalopode Todarodes pacificus Streenstrup et le Téléostéen Theragra chalcogramma (Pallas) dans le Pacifique nord. La forme adulte a été obtenue par T. Shimazu (1975) dans l'estomac d'un squale Lamna ditropis Hubbs et Follett (Lamnidae) de la mer de Bering. Description en langue japonaise, pp. 824-830, fig. 1-5, pl. I, fig. 6, œufs utérins, fig. 7-9, coupes de proglottis gravides, pl. II, fig. 10, amas de specimens adultes sur la surface de la muqueuse de l'estomac d'un Lamna ditropis H. et F. de la mer de Bering. En même temps que les Nybelinia, cet estomac contenait un Oncorhynchus nerka (Walbaum) (visible sur la figure) que T. Shimazu a considéré comme un hôte de transport (paratenic host) pour le Cestode. 


\section{E. - Chez des Crustacés}

\section{E-1. Décapodes Macroures (Penaeidae, Callianassa, Sergestes).}

(Pour des larves chez des Décapodes Macroures, voir R.-Ph. Dollfus, 1964, p. 370-373 et 1974 , p. $398-400)$.

Rafael Lamothe-Argumedo et Alejandro Cruz R. (1970, p. 379) ont rapporté au genre Parachristianella R.-Ph. Dollfus, 1946, des larves trouvées dans la glande digestive des Penaeus vannamei Boone, 1931, Penaeus stylirostris Stimpson et Penaeus californiensis Holmes, de la lagune de Urias, de la lagune de la Uva et de Bahia de Yavaros (côte nord du Pacifique mexicain). Ces larves mesuraient 1,418-2,737 $\mathrm{mm}$ de long $\times 0,338-0,724 \mathrm{~mm}$ de large.

Parachristianella monomegacantha D. N. Kruse, 1959, à l'état de plerocercus encysté, a été trouvé chez des Penaeus brasiliensis Latreille, de la Biscayne Bay (Floride), par D. L. Feigenbaum (1973, pp. 48-51, fig. 11 A-B armement des proboscis).

Parachristianella monomegacantha D. N. Kruse (1959, pp. 137-139, fig. 35-36) à l'état de plerocercus, a été trouvé dans la glande digestive avec son blastocyste pénétrant à travers la paroi de la glande, chez Penaeus duorarum M. Burkenroad, de la côte nord du golfe de Floride, par Kruse (1959).

Parachristianella dimegacantha D. N. Kruse (1959, pp. 139-140, fig. 37-40) à l'état de plerocercus, a été trouvé dans la glande digestive, avec son blastocyste, traversant en partie la paroi de la glande, chez Penaeus duorarum M. Burkenroad, à Alligator Harbor (Floride), par Kruse (1959).

Parachristianella heteromegacantha D. L. Feigenbaum 1973, à l'état de plerocercus enkysté, a été trouvé chez des Penaeus brasiliensis Latreille, de la Biscayne Bay (Floride), par D. L. Feigenbaum (1973, pp. 51-55, fig. 12-14, specimen entier et armement des proboscis).

Prochristianella penaei D. N. Kruse (1959, pp. 154-157, fig. 28-34), à l'état de plerocercus enkysté a été trouvé par Kruse (1959) dans la glande digestive et tissus environnants, ainsi que dans l'estomac; les blastocystes pénétraient fréquemment dans la paroi de la glande digestive, chez Penaeus aztecus Ives, Penaeus duorarum M. Burkenroad et Penaeus setiferus L. de la côte nord du golfe de Floride.

Prochristianella penaei D. N. Kruse 1959, à l'état de plerocercus enkysté, a été trouvé chez des Penaeus brasiliensis Latreille, de la Biscayne Bay (Floride), par D. L. Feigenbaum (1973, pp. 55-57, fig. 15 A-B, armement des proboscis).

Renibulbus penaeus D. L. Feigenbaum (1973, pp. 58-62, fig. 16 A-18-B, spécimen entier et armement des proboscis), à l'état de plerocercus enkysté, a été trouvé chez des Penaeus brasiliensis Latreille de la Biscayne Bay (Floride) par D. L. Feigenbaum, qui a créé pour cette larve une nouvelle famille: Renibulbidae et un nouveau genre 
Renibulbus, où l'armement métabasal des proboscis est hétératanthe hétéromorphe, comme chez d'autres Heteracantha typica, mais dont les bulbes sont très courts et réniformes.

La larve énigmatique, dubitativement attribuée aux Lecanicephalidae (4), par R. F. Hutton, F. Sogandares-Bernal, B. Eldred, R. M. Ingle et K. O. Woodburn (1959), souvent retrouvée chez divers crustacés de Floride (voir R.-Ph. Dollfus, 1964, pp. 371372 ; 1974, pp. 398-399), a été retrouvée aussi par D. L. Feigenbaum (1973, pp. 62-63) chez des Penaeus brasiliensis Latreille de la baie de Biscayne (Floride). Il a remarqué que cette larve piriforme, à ventouse apicale éversible, localisée dans la paroi interne de l'intestin moyen, peut être présente en grand nombre, de 5 à 226 , dans le même individu hôte. Souvent, dit Feigenbaum, 2 ou 3 individus sont attachés entre eux, formant une sorte de chaîne. Les specimens en extension, sur le vivant, mesurent 143 à $215 \mathrm{~mm}$, avec une largeur de 57 à $43 \mathrm{~mm}$. Les 7 spécimens fixés mesurent 102 (66-132) de long sur 51 (37-61) de large; la ventouse apicale 28 (23-35) de long sur 39 (28-50) de large.

Larve indéterminée de Cestode. Cette larve a été trouvée par D. N. Kruse (1959, p. 140) dans la limitante interne de l'intestin moyen de Penaeus aztecus Ives et Penaeus duorarum M. Burkenroad, d'Alligator Harbor et Apalachicola Bay (Floride). Cette larve au corps piriforme $(0,125 \times 0,066 \mathrm{~mm})$, à ventouse apicale bien développée $(0,033 \times 0,046)$, est pourvue de quatre glandes immédiatement postérieures à la ventouse apicale. Le canal évacuateur de chaque glande traverse la paroi de la ventouse et s'ouvre dans la cavité de la ventouse. La cuticule du corps porte des rangées serrées de fines épines. D. N. Kruse a donné une description détaillée de cette larve, dont la position systématique est incertaine, mais qui a été tentativement rapportée aux Lecanicephalidae par R. F. Hutton et collab. (1959, pp. 22-24, fig. 33-34). Voir, au sujet de cette larve, R.-Ph. Dollfus, 1964, pp. 371-372; 1974, pp. 398-399. Elle a été retrouvée aussi par David L. Feigenbaum (1973, pp. 62-63).

A. Cruz-Reyes $(1974$, p. 1729) a étudié l'helminthofaune de trois espèces de Penaeus de la côte nord-ouest du Mexique $\left(20^{\circ} 50-26^{\circ} 45 \mathrm{~N}\right.$ et $106^{\circ} 20-109^{\circ} 50$ W). Quelles étaient ces trois espèces ? Cela n'est pas précisé. Tous les individus parasités par des larves de Cestodes présentaient des déformations du céphalothorax et de la glande digestive ; les tissus contenant les plerocercus étaient d'une couleur blanc crème et sans consistance, en outre, il y avait une « obstruction of other organs ».

Quels étaient ces Cestodes? Cruz-Reyes ne le dit pas, il mentionne seulement leur appartenance aux Trypanorhyncha.

Chez les Callianassa g. gigas Dana, 1852, de la Californie du Sud, Michael L. Heinz et Murray D. Dailey (1974, p. 168) ont trouvé la larve d'Eutetrarhynchus schmidti Heinz et Dailey, adulte dans la valvule spirale de Urolophus halleri Cooper et Rhinobatos productus (Ayres) d'Anheim Bay et Seal Beach (Californie). Cet adulte mesure, à maturité, $8,96 \mathrm{~mm}$ de long, au maximum.

(4) L'attribution par Hutton (1962) au genre Polycephalus = Parataenia n'est peut-être pas définitive. Le genre Polypocephalus a été révisé par C. K. Subhapradha (1951). 
Cette larve est à comparer avec celle trouvée par R. T. Young (1954, p. 110) dont il a été question dans R.-Ph. Dollfus, 1964, p. 373.

A. J. Slankis et G. G. Shevchenko (1974, p. 134) mentionnent la présence de larves de Tétrarhynques chez Sergestes lucens Hansen, Sergestes sp. du plancton du Pacifique tropical occidental.

\section{E-2. Chez les Décapodes Brachyoures.}

Christian P. Vivares (1973 $a$, p. 203) a trouvé des Tétraphyllides:

$1^{\circ}$ du type Phyllobothriidae chez Macropipus depurator (L. 1758) et Goneplax rhomboides (L. 1758) du golfe du Lion;

$2^{\circ}$ du type Echeneibothrium chez Macropipus depurator (L. 1758) du golfe du Lion ;

$3^{\circ}$ du type Onchobothriidae chez Xantho incisus granulicarpus (J. Forest, 1953) du golfe du Lion;

$4^{\circ}$ d'un type indéterminé chez Pinnotheres pinnotheres (L. 1758), du golfe du Lion.

Une espèce de Tétrarhynque: Eutetrarhynchus ruficollis (Eysenhardt, 1829) chez Macropipus depurator (L. 1758), du golfe du Lion.

Une espèce de Diphylle: Echinobothrium affine Diesing, 1863, chez Ethusa mascarone (Herbst, 1785), du golfe du Lion.

C.-P. Vivares $(1973 \mathrm{~b}$, p. 662) relate avoir trouvé, dans la région de Sète et le golfe de Tunis, chez Macropipus depurator (L. 1758), dans les caeca hépatiques et la cavité générale, des Scolex polymorphus O.F.M.; il mentionne aussi, chez le même hôte, dans les mêmes régions, Eutetrarhynchus ruficollis (Eysenhardt, 1829).

C.-P. Vivares $(1973$ b, p. 663) a trouvé Scolex polymorphus O.F.M. dans le caecum digestif de Xantho incisus granulicarpus (J. Forest, 1953) et Pinnotheres pinnotheres (L. 1758), aussi dans le caecum hépatique de Goneplax rhomboides (L. 1758) et de Maja squinado (Herbst, 1788), à Sète (Hérault).

\section{E-3. Edriophthalmes Amphipodes.}

Les larves de Bothrimonus sturionis G. L. Duvernoy, 1842, attribuées tantôt au genre Bothrimonus Duvernoy, 1842, tantôt au genre Diplocotyle H. Krabbe, 1874 (actuellement considéré comme synonyme) signalées chez des Amphipodes ont été observées principalement chez des Gammaridae (genres Gammarus et Marinogammarus), toutefois A.V. Uspenskaya (1955, 1960 et 1963), ainsi que je l'ai rappelé précédemment (Ann. Parasitol. hum. comp., 1964, 39, 367) en a trouvé chez Anonyx fugax (Phipps), fam. Lysianassidae en mer de Barents. Chez une autre espèce de Lysianassidae: Psammonyx nobilis (Stimpson, 1853) de Terre-Neuve et de la Nouvelle-Angleterre (plusieurs localités de la côte du Maine). K. J. Scott et W. L. Bullock (1974, pp. 256-257) ont récolté la larve de Bothrimonus sturionis G. L. Duvernoy. Ces auteurs ont noté, pour chaque localité, le nombre de Psammonyx 
parasités selon le mois de l'année. D’après leurs observations, les œufs du Bothrimonus sont ingérés par les Psammonyx pendant l'été et, après avoir mué, la larve continue à grandir. Lorsqu'elle a achevé sa croissance, elle pourra poursuivre le cycle évolutif si l'Amphipode est ingéré par un poisson vivant sur le fond, tel que Pseudopleuronectes americanus (Walbaum).

Les ơ de Psammonyx infestés développent normalement leurs gonades, mais pas les + .

\section{E-4. Entomostraces Copépodes.}

K. H. Rao et R. Madhavi (1966, pp. 70-71, fig. 1), dans le plancton de la Baie de Bengale, ont trouvé un Copépode, Eucalanus subcrassus Giesbrecht, 1888, fortement infesté par une larve non enkystée de Scolex polymorphus du type unilocularis; celle-ci contenait 3 grands et 2 petits corpuscules calcaires; son système osmorégulateur était bien développé et comprenait 4 ampoules à flamme vibratile (fig. 1).

Fig. 1. - Copie de la fig. 1, p. 71, de K. Hamumontha Rao et $\mathrm{R}$. Madhavi FC ampoule à flamme vibratile; $\mathrm{L}$ canal longitudinal ; $\mathrm{T}$ canal transversal ; V vessie - $\mathrm{M}$ tronc excréteur médian.

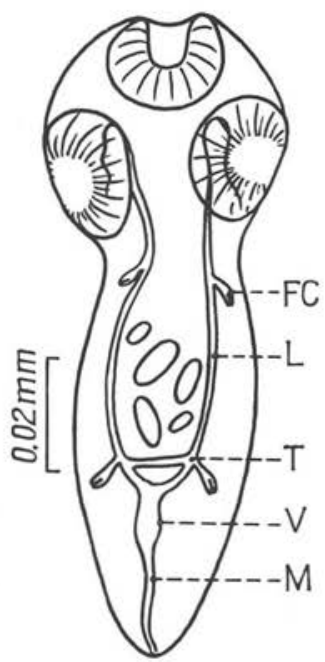

\section{E-5. Schizopoda Euphausiacea.}

A. J. Slankis et G. G. Shevchenko (1974, p. 134) mentionnent la présence de larves de Cestodes chez divers Euphausiacea du plancton du Pacifique tropical occidental: Euphausia similis Sars, Euphausia diomedea Ortmann, Euphausia brevis Hansen, Euphausia sp., Thysanopoda tricuspidata H. M. Edwards, Thysanopoda sp.

Chez Euphausia diomedea Orthmann, sur 22 larves de Cestodes, 21 étaient des larves de Tétrarhynques.

En deux publications, Takeshi Shimazu (1975) a traité des larves de Cestodes trouvées chez des Euphausiidae du Pacifique nord, mer de Bering et côte nord du Japon. Dans la première de ces publications, T. Shimazu (1975, pp. 814-815, fig. 1-4) a décrit, comme plérocercoïde, la postlarve de Nybelinia surmenicola Yô K. Okada in R.-Ph. Dollfus, 1929, qu'il a identifiée chez Euphausia pacifica Hansen, Thysanoëssa longipes Brandt, Thysanoëssa inermis (Kröyer), Thysanoëssa naschii (M. Sars) et une quatrième espèce non identifiée.

T. Shimazu (ibid., pp. 815-816, fig. 5) a décrit comme plérocercoïde de Pelichnibothrium caudatum Zschokke et Heitz, 1914, une larve du type Scolex pleuronectis O.F.M. parasitant Thysanoëssa longipes Brandt. 
T. Shimazu (ibid., pp. 815-816, fig. 6-7) a décrit comme cysticercoïde d'un « unidentified cestode » une larve qui, dit-il, ressemble à Anomotaenia larina (Krabbe, 1869), (Cyclophyllidea Dilepididae), parasitant Thysanoëssa inermis (Kröyer) (5).

T. Shimazu (1975, pp. 815-816, fig. 8, crochet du rostre) a décrit comme cysticercoïde d'un « unidentified Cestode », une larve qui, dit-il, ressemble à Anomotaenia armillaris (Rudolphi, 1810) parasitant Thysanoëssa inermis (Kröyer) (6).

La deuxième publication concerne seulement des larves de Cestodes parasitant des Euphausia similis Sars de Suruga Bay. Ces larves ont été décrites dans l'ordre ci-après par T. Shimazu (1975) :

a) pp. 122-123, 128, fig. 1-3 : larve à longue queue d'un Diphyllide, ressemblant à Echinobothrium acanthinophyllum G. Rees, 1961, par le nombre, la disposition, la forme et les dimensions des crochets du rostre;

b) pp. 123-125, fig. 4-7: larve d'Eutetrarhynchidae gen. sp., enclose dans son blastocyste (7) ;

c) pp. 124-125, fig. 8-11: Tetrarhynchobothrium sp.; la larve de la fig. 8 est encore contenue dans son blastocyste;

d) pp. 125-126, 128, fig. 12-14: «much branched cysticercoid-like larvae » qui, par production endogène, donne un grand nombre de larves enkystées d'un Cestode indéterminé. Ces larves, de forme ovale, ont quatre bothridies circulaires situées dans leur tiers antérieur et une petite ventouse apicale; elles rappellent donc morphologiquement quelques formes du groupe Scolex pleuronectis O.F.M. ;

e) pp. 126, 127, 128, fig. 15 ; «plerocercoid like larva d'un Cestode inconnu. D'après la figure, il s'agit d'une larve de forme régulièrement ovale, dont la paroi externe consiste en une épaisse enveloppe ciliée.

Les descriptions de toutes ces larves sont, malheureusement, publiées en langue japonaise. Un «abstract» en langue anglaise ne donne que des renseignements très sommaires sur la morphologie de cet important matériel.

\section{Bibliographie}

AldRICH (D. V.), 1964. - Observations on the ecology and life cycle of Prochristianella penaei Kruse (Cestoda: Trypanorhyncha). J. Parasit., 50 (Section 2), 30.

Aldrich (D. V.), 1965. - Idem. J. Parasit., 51, 370-376, fig. 1-4.

AZARIAH (J.), 1968. - Occurrence of a trypanorhynchan larva in Amphioxus (Branchiostoma lanceolatum). Current Science, 37, 439-440, fig. 1-2.

Cruz-Reyes (A.), 1974. - Helminthofauna of schrimp of the genus Penaeus Fabricius, from north west of Mexico. Third internat. Congress. of Parasitol., München, 25-31 Aug., 1728-1729.

(5) L'adulte a été décrit chez des Oiseaux, Larus, Rissa.

(6) L'hôte-type de l'adulte est Alca pica (Bonnaterre).

(7) Des larves ayant une structure voisine (où la pars postica scolecis est attenante par un prolongement musculaire, à la paroi interne du blastocyste) ont été figurées dans $\mathrm{R}$.-Ph. Dollfus 1967, p. $156-161$, fig. $1-8$. 
Dollfus (R.-Ph.), 1964. - Enumération des Cestodes du plancton et des Invertébrés marins (6 $6^{e}$ contribution). Ann. Parasit. hum. comp., 34, 329-379, 1967. Idem ( $7^{\circ}$ contribution). Ann. Parasit. hum. comp., 42, 155-178, 1974. Idem ( $8^{\circ}$ contribution). Ann. Parasit. hum. comp., 49, 381-410.

Feigenbaum (D. L.), 1973. - Parasites of the commercial shrimp, Penaeus vannami Boone and Penaeus brasiliensis Latreille. Thesis Univ. Miami... Master of Science, VII +85 p., fig. 1-20.

Hamilton (K. A.) and Byram (J. E.), 1974. - Tapeworm development : the effects of urea on a larval Tetraphyllidean. J. Parasit., 60, 20-28, fig. 1-20.

Heinz (M. L.) and Dalley (M. D.), 1974. - The Trypanorhyncha (Cestoda) of Elasmobranch fishes from southern California and northern Mexico. Proc. helminthol. Soc. Washington, 41, 161-169. fig. 1-28.

Lamothe Argumedo (R.) y Cruz R. (A.), 1970. - Informe sobre los parasitos del Camaron, en relacion con los planes piloto escuinapa y yavaros, pp. 372-383, In « Informe final de los trabajos contratados en los planes piloto EscuinapaYavaros ». Univ. nacion. autonom. Mexico D.F., 409 p.

Quick (J. A. jr), 1971. - Pathological and parasitological effects of elevated temperatures on oysters with emphasis on Labyrinthomyxa marina. In : A preliminary investigation: the effect of elevated temperatures on the american oysters, Crassostrea virginica (Gmelin). A Symposium edited by J. A. Quick, Florida Dept. natural Resources. Profession. Papers Series. Marine Research Laboratory, St-Petersburg, Florida, pp. 105-176, fig. V1-V22.

Rao (K. H.) and Madhavi (R.), 1966. - Tetraphyllidean larva (Cestoda) in the Copedod Eucalanus subcrassus Giesbrecht 1888 of Waltair coast, Bay of Bengal. Current Science, 35, 70-71, fig. 1 .

Reimer (L.W.), 1974. - The position of Cephalopods in Life cycles of Helminths of marine fishes. Third internat. Congress of Parasitol., München 25-31 Aug., 17271728.

Reimer (L. W.), 1975. - Cestodenlarven in Wirbellosen der Küste von Madras. Angewandte Parasitologie, Jena, 16, 2-16, fig. 1-14.

RIFKIN (E.), 1969. - The internal defense mechanisms of the oyster Crassostrea virginica in response to the Cestode Tylocephalum sp. Dissertation Abstracts 30 B, 4432-B.

RIFKIN (E.) and CHENG (T. C.), 1969. - The fine structure of the tegument and associated structures of Tylocephalum metacestodes. Transact. amer. microsc. Soc., 88, 166167 (Abstracts presented at 84th annual meeting, sept. 1968).

Scott (K. J.) and Bullock (W. L.), 1974. - Psammonyx nobilis (Amphipoda: Lysianassidae), a new host for Bothrimonus sturionis (Cestoda: Pseudophyllidae). Proc. helminthol. Soc. Washington, 41, 256-257.

Shimazu (T.), 1975. - On the parasitic organisms in a krill, Euphausia similis from Suruga Bay. V. Larval cestodes. Japan. Journ. Parasit., 24, 122-128, fig. 1-15.

Shimazu (T.), 1975. - Some Cestode and Acanthocephalan larvae from Euphausid crustaceans collected in the northern North-Pacific Ocean. Bull. japan. Soc. scient. Fisheries, 41, 813-821, fig. 1-12.

Shimazu (T.), 1975. - A description of the adult of Nybelinia surmenicola with discussion on its life-history (Cestoda: Trypanorhyncha: Tentaculariidae). Bull. japan. Soc. scient. Fisheries, 41, 823-830, fig. 1-10. 
Slankis (A. J.) and Shevchenko (G. G.), 1974. - Some data on the infestion of the plancton invertebrates with helminth larvae in the west part of the tropical zone of the Pacific. Transact. Pacific. Res. Inst. Fisheries and Oceanogr. (Tinro), Vladivostok, 88, 129-138, fig. 1-16.

SubHapradHa (C. K.), 1948. - Helminth parasites of the economic fishes of the Madras coast. Mast. Sc. Thesis Univ. of Madras (non publiée).

Subhapradha (C. K.), 1951. - On the genus Polypocephalus Braun, 1878 (Cestoda) together with description of six new species from Madras. Proc. zool. Soc. London, 121, part. II, 205-235, fig. 1-34; pl. I.

ViVARES (C.-P.), 1973 a. - Le parasitisme chez les Brachyoures (Crustacea, Decapoda) de la côte méditerranéenne française et des étangs du Languedoc-Roussillon. Vie et Milieu, 23, fasc. 2, sér. A, 191-218, fig. 1-8.

Vivares (C.-P.), 1973 b. - Etude du parasitisme des Crustacés Décapodes Brachyoures en Méditerranée occidentale: premiers résultats. Rapport Commission internat. Mer Médit., Copenhague, 21, 661-663.

\section{ERRATA ET CORRIGENDA}

- Dans ma note «Sur le cycle évolutif d'un Cestode Diphylle », Ann. Parasit. hum. comp., $1964,39, \mathrm{n}^{\circ} 3$, p. 236 , au lieu de lateriporum, lire lateroporum.

- Dans ma $6^{\circ}$ Contribution (1964) p. 338, 17 ligne au lieu de tropa, lire trapa ; $18^{\circ}$ ligne, au lieu de Anataraman, lire Anantaraman.

La page numérotée 342 par erreur est, en réalité la page 343 et la page numérotée 343 est en réalité la page 342 .

La dernière ligne de la page 363 est à transférer comme dernière ligne page 365 et la dernière ligne de la page 365 est en réalité la dernière ligne de la page 363 .

- Dans ma $8^{\circ}$ Contribution (1974), p. 409, ligne 25, au lieu de décrit, lire écrit. 